\title{
Reflets
}

Revue ontaroise d'intervention sociale et communautaire

\section{Des jeunes à l'écart du social?}

Histoires des jeunes francophones sans abri à Ottawa-Carleton

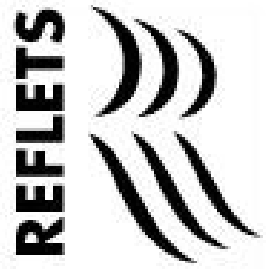

\section{Marc Molgat et Denise Lemire}

Volume 1, numéro 1, printemps 1995

Des pratiques à notre image : défis et ressources

URI : https://id.erudit.org/iderudit/026058ar

DOI : https://doi.org/10.7202/026058ar

Aller au sommaire du numéro

Éditeur(s)

Reflets : Revue ontaroise d'intervention sociale et communautaire

ISSN

1203-4576 (imprimé)

1712-8498 (numérique)

Découvrir la revue

Citer cet article

Molgat, M. \& Lemire, D. (1995). Des jeunes à l'écart du social? Histoires des jeunes francophones sans abri à Ottawa-Carleton. Reflets, 1(1), 141-163.

https://doi.org/10.7202/026058ar

Tous droits réservés (C) Reflets : Revue ontaroise d'intervention sociale et communautaire, 1995
Ce document est protégé par la loi sur le droit d'auteur. L'utilisation des services d'Érudit (y compris la reproduction) est assujettie à sa politique d'utilisation que vous pouvez consulter en ligne.

https://apropos.erudit.org/fr/usagers/politique-dutilisation/ 


\title{
Des jeunes à l'écart du social?
}

\section{Histoires des jeunes francophones sans abri à Ottawa-Carleton}

En basant sa recherche sur une théorie de l'exclusion sociale, l'auteur étudie la situation de jeunes francophones sans-abri dans Ottawa-Carleton. Il a questionné ces jeunes sur leur identité franco-ontarienne et a exploré les stratégies qu'ils mettent en oeuvre pour survivre en français dans un contexte de précarité. De l'absence de soutien institutionnel et de services en français découlent plusieurs questions, tant au niveau de l'intervention que des politiques sociales.

\author{
Marc Molgat \\ Étudiant au programme de doctorat en sciences humaines appliquées, Université \\ de Montréal \\ avec la collaboration de
}

\section{Denise Lemive}

Consultante en recherche sociale à la firme Beaulieu et Lemire Consultation

\section{Introduction}

Un rapide coup d'oeil dans le secteur du Marché-By à Ottawa confirme l'émergence suite aux crises économiques des années 1980 et 1990, d'une nouvelle pauvreté, soit celle des jeunes sans abri. Au rythme des années, les rangs de l'itinérance se sont agrandis et diversifiés. Ce ne sont pas, aujourd'hui, que de vieux hommes alcooliques qui vous quêtent quelques sous; ce sont aussi des femmes violentées, des personnes ayant des problèmes psychiatriques, des gens appartenant à diverses communautés ethniques et aussi des jeunes. Pour Simard (1990:3), le concept de sans-toit 
Reflets

englobe diverses réalités: errant, gitan, vagabond, bohémien, clochard, sans-abri, robineux, nomade, itinérant. Cette terminologie laisse supposer un choix de vie, de la part des personnes qu'elle décrit, occultant de ce fait la question d'exclusion sociale liée aux problèmes de chômage, de faible revenu, de sous-scolarisation, l'absence de logement et de santé physique et mentale. La définition de Lise Lalonde (1993:63) s'inscrit dans le schème de pensée qui fait du sans abri un sujet social:

....(elle) représente une problématique propre aux années 1980 et après, comprenant un nombre important et sans cesse croissant de personnes socialement marginalisées et isolées, dont le revenu est au-dessous du seuil de la pauvreté, pour qui la santé physique et mentale est précaire, et dont la trajectoire spatiale (comprenant les formes de logement et la fréquentation des lieux) est diversifiée. Ces personnes font partie d'un groupe fort hétérogène en termes de caractéristiques sociodémographiques, de besoins, de durée de l'itinérance et de liens aux services.

L'analyse de la problématique de l'itinérance des jeunes francophones dans la région d'Ottawa-Carleton s'inscrit dans ce cadre. Le problème des jeunes sans abri n'est donc pas uniquement lié aux dysfonctions attribuées à la personne (Brennan, Huizinga et Elliot, 1978; Roberts, 1981; Huber, 1987; Conseil de planification sociale, 1989; Whitbeck et Simons, 1990; Community Task Force on Homeless/Street Youth, 1992; Bernier, Morissette et Roy, 1992) mais est aussi tributaire d'une problématisation socioéconomique (Comité consultatif du ministère sur l'année internationale du logment des sans-abri,1987; Lamontagne et al.,1987; Bégin, 1992; Lalonde, 1993).

Dans cet article, nous examinons l'itinérance des jeunes, non pas comme problème extérieur à la société mais bien comme phénomène qui y est intimement lié. Les interrogations principales que nous abordons sont les suivantes: Quelles perceptions les jeunes francophones sans abri ont-ils de leur situation 
d'itinérance et de leur statut de Franco-Ontariennes ou FrancoOntariens? Quelles stratégies ces jeunes mettent-ils en oeuvre pour lutter contre l'itinérance et dans quelle mesure ces stratégies s'appuient-elles sur une identité franco-ontarienne?

\section{D'où vient la notion de jeunesse?}

"...la jeunesse est

devenue une étape de transition entre le monde de l'enfance et celui de l'adulte où les jeunes ne sont ni adultes ni enfants...»
C'est sous l'influence des moralistes au XV $\mathrm{XV}^{\mathrm{e}}$ siècle que l'on commence à fonder des ordres enseignants destinés à donner une éducation morale aux enfants (Ariès, 1973). Le mouvement vers l'éducation institutionnelle des enfants se double d'un mouvement de fond vers le développement du sentiment de famille et l'érection d'un mur autour de la vie privée (Ariès, 1973). Les conséquences de ces mouvements sur les liens de socialité se font toujours ressentir aujourd'hui; l'isolement des jeunes par rapport au voisinage et aux relations avec d'autres adultes a comme conséquence de les empêcher de trouver des appuis et des solutions de rechange quand des pressions trop fortes pèsent sur eux dans leur foyer. Aujourd'hui, la jeunesse est devenue une étape de transition entre le monde de l'enfance et celui de l'adulte où les jeunes ne sont ni adultes ni enfants, ou comme Bourdieu (1980:145) l'indique «adultes pour certaines choses et enfants pour d'autres».

L'image du concept de pauvreté, plus ou moins positive parce qu'associée à celle du Christ, selon Gutton (1974:94), qui recoupe à la fois mendicité et vagabondage, se transforme au cours des siècles. Les lois deviennent plus répressives et contrôlantes et l'État prend en charge les mineurs (Meyer, 1977:36). Par exemple, en 1810, la loi française ordonne que les personnes mineures de moins de 16 ans qui mendient ou vagabondent soient replacées dans leurs familles ou conduites dans une maison de correction (Meyer, 1977:36-37) pour fin de rééducation ou pour la mise au travail. Aujourd'hui, au Canada, les pratiques sont à peu près semblables: les enfants de moins de 16 ans qui se font prendre à 
Reflets

mendier ou à vagabonder sont placés dans des organismes d'aide à l'enfance (Ursel, 1992:112-114). Le meilleur intérêt des enfants prime dans la législation ontarienne, La loi sur les services à l'enfance et à la famille (Reitsma-Street, 1990:519). Par contre, comme cette loi ne s'applique pas à eux, les jeunes sans abri de plus de 16 ans ne sont pas pris en charge par l'État de la même façon. En Ontario et dans la région d'Ottawa-Carleton en particulier, les services offerts à ces jeunes sans abri sont plutôt semblables à ceux de dépannage d'urgence dispensés aux adultes (abris, soupes populaires, banques d'alimentation et haltes-accueil).

\section{Les causes del'itinérance des jeunes}

De nombreux auteurs ont discuté des causes de l'itinérance des jeunes en général. Par exemple, Côté (1991:73 et 150) affirme que c'est la violence et l'abandon qui caractérisent les parents tyranniques des jeunes qu'elle a interviewés. D'autres auteures et auteurs insistent à leur tour sur des facteurs liés aux $d y$ sfonctions familiales: mauvaises pratiques de parentage et fréquence de la violence (Whitbeck et Simons, 1990:109); abus sexuel et physique au foyer conjugués aux problèmes personnels et scolaires des jeunes (Kurtz, Jarvis et Kurtz,1991); mort, divorce, abus physique, maladie, hospitalisation ou alcoolisme des parents (Roberts, 1981); manque de relations affectives entre parents et enfants (Brennan, Huizinga et Elliott,1978); dégradation de la situation familiale résultant du prolongement de la cohabitation entre parents et enfants (Huber, 1987). Enfin, les études locales du Conseil de planification sociale d'Ottawa-Carleton (1989) et du Community Task Force on Homeless/Street Youth (1992) posent avant tout les problèmes de violence physique et sexuelle au foyer comme les causes expliquant la fuite des jeunes. Même si la famille peut être un milieu anxiogène, violent et malsain, il ne faut cependant pas en rester là dans l'analyse de l'itinérance des jeunes. 


\section{L'exclusion sociale comme approche théoriqueà l'itinérance desjeunesfrancophones}

Une approche s'articulant autour des processus d'exclusion (Xiberras, 1993; Castel, 1991, Strohl et Xiberras, 1992) nous permet d'appréhender les problèmes socio-familiaux et structurels qui mènent à l'itinérance et qui rendent problématique l'intégration socio-économique des jeunes sans abri. Cette approche contribue aussi à l'élaboration de pistes d'interprétation et d'analyse tenant compte du poids de la culture dominante sur l'identité franco-ontarienne, des problèmes socio-économiques des jeunes francophones, du vécu des jeunes itinérants et des politiques et services mis en oeuvre pour répondre à ce phénomène.

Maintenir et développer une identité franco-ontarienne n'est pas facile pour plusieurs jeunes car ce statut porte en lui des filières importantes d'exclusion sociale dont celles liées aux problèmes de scolarisation et d'emploi. C'est sous l'angle d'analyse du poids de la culture dominante qu'il devient évident que la construction et la préservation d'un sens d'appartenance franco-ontarien sont des réalités difficiles à vivre, en particulier lorsqu'on est jeune et sans abri. En effet, la culture populaire nord-américaine et anglophone est envahissante et touche de près les Franco-Ontariennes et Franco-Ontariens. Comme le développement de leur identité est affecté par l'omniprésence de cette culture dominante, les jeunes pourraient être portés à s'écarter d'une identité francoontarienne. De surcroît, ce désir d'éloignement pourrait se faire plus pressant lorsqu'ils sont confrontés à un environnement familial suffocant et à des relations socio-familiales qui les pous-

«...l'identité francoontarienne peut représenter une oppression pour les jeunes, rendant ainsi la culture dominante plus attirante et sécuritaire.» sent à fuir. En somme, l'identité franco-ontarienne peut représenter une oppression pour les jeunes, rendant ainsi la culture dominante plus attirante et sécuritaire.

Les municipalités ontariennes, qui sont en grande partie responsables des services sociaux, ne sont pas tenues de respecter la Loi de 1986 sur les services en français (Loi 8); l'offre de services en français peut alors demeurer faible ou même inexistante. Nous devons nous attarder à l'impact de cette pénurie de services en français sur les jeunes, en particulier pour celles et ceux qui sont 
Reflets

"...les jeunes francophones auront tendance à se construire une identité bilingue ou anglophone afin d'éviter un plus grand isolement.» sans abri. En effet, puisque les programmes offerts par les organismes communautaires d'Ottawa-Carleton se déroulent presque exclusivement en anglais - comme à la Halte-accueil du centreville du Bureau des services à la jeunesse (BSJ) et à l'Abri d'urgence pour jeunes de l'Armée du Salut - les jeunes francophones auront tendance à se construire une identité bilingue ou anglophone afin d'éviter un plus grand isolement. S'intégrer à l'espace linguistique et social des organismes, avoir accès à des programmes favorisant leur intégration sociale et économique et créer des liens avec les autres jeunes qui vivent les mêmes problèmes deviennent en ce sens des stratégies pour éviter l'isolement, voire l'exclusion. Ces stratégies passent souvent par l'adoption d'une identité bilingue, anglophone et, en ce sens, témoignent du rôle puissant d'assimilation et de non-reconnaissance de l'identité des organismes communautaires anglophones ou dits bilingues. Conjugée à l'oppression que peuvent vivre les jeunes francophones sans abri et aux filières de marginalisation associées à l'identité franco-ontarienne, l'absence d'espaces ou de milieux francophones adaptés aux besoins de ces jeunes favorise leur assimilation.

\section{Méthodologie de la recherche}

Une méthode de recherche qualitative, axée sur des entrevues semidirigées auprès de jeunes francophones sans abri a été utilisée afin de rendre compte de la complexité de leur situation et de la richesse de leurs discours. La recherche visait à présenter les jeunes interviewés en tant que sujets qui réfléchissent sur leur situation et qui agissent sur celle-ci en fonction des outils et des occasions qui leur sont disponibles. Comme la situation de l'itinérance des jeunes est complexe et mouvante, la souplesse de la méthode qualitative en fait une méthode de choix (Deslauriers, 1991:14).

Un échantillon non probabiliste restreint a été choisi pour la recherche: nous prévoyions interviewer six ou sept jeunes FrancoOntariennes et Franco-Ontariens sans abri (ou l'ayant été dans la 
dernière année) entre 16 et 24 ans. Dans un premier temps, le recrutement des jeunes s'est effectué à partir d'une méthode d'échantillonnage dite de boule de neige (Beaud, 1984:187 cité dans Mayer et Ouellet, 1991:390). Deuxièmement, la méthode de l'échantillon volontaire (Beaud, 1992) a été exploitée afin de rejoindre les jeunes qui ne sont pas connus par des personnes oeuvrant au sein d'organismes. Des annonces de recrutement ont été placées dans des haltes-accueil et des abris d'urgence. Par ces stratégies, neuf jeunes de moins de 21 ans ont accepté de participer à une entrevue semi-dirigée. Les entrevues ont été enregistrées; elles ont duré entre une heure et demie et deux heures et demie et se sont déroulées sur le terrain, à la convenance des jeunes et souvent de façon imprévue. Les entrevues ont été codées : une grille a été préparée pour chacune des catégories d'analyse; les noyaux de sens et les citations les accompagnant y ont été placés, selon les différentes personnes interviewées. Une fois ce squelette bâti, une comparaison horizontale a été réalisée entre les discours des jeunes selon les thèmes établis. De cette analyse comparative se sont dégagées des tendances et des divergences en fonction desquelles des propositions d'analyse ont été retenues.

L'implication de groupes communautaires dans le cadre de deux réunions de consultation a permis une certaine sensibilisation face à l'itinérance des jeunes Franco-Ontariennes et FrancoOntariens et nous a donné l'occasion de retravailler les résultats de la recherche (Mayer et Ouellet, 1991:173).

\section{Résultats de la recherche: analyse descriptive des données}

Les caractéristiques socio-démographiques desjeunes

Cinq femmes et quatre hommes, dont les âges variaient de 16 à 21 ans, ont été interviewés. Alors que les femmes avaient 16, 16, 
Reflets

18, 18, et 21 ans respectivement, les hommes avaient 17, 20, 20 et 21 ans. Ainsi, les femmes interviewées étaient plus jeunes que les hommes, ce qui reflète les tendances du profil âge et sexe d'autres études récentes (Kufeldt et Burrows, 1994; Community Task Force, 1992; Conseil de planification d'Ottawa-Carleton, 1989). Tous les jeunes peuvent être considérés d'origine ethnique canadienne-française quoique trois jeunes ont défini leur origine ethnique comme étant mixte selon les configurations suivantes: canadienne française et indienne, canadienne française et italienne, canadienne française et française/africaine. Deux jeunes ont défini leur origine ethnique plus spécifiquement comme étant franco-ontarienne et acadienne. Quant à leur lieu de naissance, cinq jeunes sont nés dans l'est de l'Ontario, deux dans l'Outaouais québécois, une à Québec et un en Nouvelle-Écosse. Tous les jeunes vivent dans la région d'Ottawa-Carleton depuis au moins deux ans, et six y ont vécu pendant sept ans ou plus.

Tous les jeunes étaient sans abri au moment de l'entrevue ou l'avaient été dans la dernière année. Lors des entrevues, deux des cinq femmes étaient logées dans un abri d'urgence et venaient de vivre une séparation avec le chum avec qui elles avaient habité en appartement. Un homme et une autre femme étaient dans un abri d'urgence. Les autres vivaient dans les habitations suivantes: maison de chambre (2), appartement avec amis/conjointe (2), maison avec parents (1). Donc, parmi les jeunes interviewés, quatre avaient une situation de logement extrêmement précaire (abri d'urgence), deux avaient une situation de logement instable (maison de chambre) et trois partageaient divers arrangements de logement. De plus, les jeunes étaient célibataires et sans enfant au moment de l'entrevue, sauf pour un jeune homme qui habitait en appartement avec sa conjointe et leur enfant d'un an.

Sans exception, les jeunes avaient le français comme langue maternelle. Un jeune homme a affirmé avoir à la fois le français et l'anglais comme langues maternelles. Au niveau de la langue d'usage par contre, les jeunes ont avoué qu'ils utilisaient surtout l'anglais, sauf pour une jeune femme et un jeune homme. Tous les jeunes ont affirmé avoir perdu leur capacité de bien parler le français après être devenus sans abri. 


\section{Les histoires de vie des jeunes}

\section{Leurs familles}

Les histoires de vie des jeunes sont marquées par des relations familiales très difficiles. Sauf pour deux jeunes, tous ont vu leurs parents divorcer, se séparer ou reconstituer leurs familles avec de nouvelles ou de nouveaux partenaires. Dans ces situations, ce sont les mères qui s'étaient retrouvées responsables des enfants et, dans presque tous les cas, elles ont repris une vie de couple avec un nouveau mari ou copain qui a souvent été mal accepté par les jeunes, parce qu'il était violent. Les jeunes dont la mère n'a pas repris une vie de couple après la séparation ou le divorce ont connu une situation économique familiale particulièrement difficile. Cependant, la situation économique ne semble pas constituer un fil conducteur dans les trajectoires des jeunes. L'alcoolisme des pères revient par contre souvent dans les histoires de

"C'est avec colère et déception que ces jeunes parlent généralement de leur père.» vie. C'est avec colère et déception que ces jeunes parlent généralement de leur père. Tous les jeunes interviewés ont été victimes d'abus physique, psychologique ou sexuel de la part des proches.

Les déménagements constants auxquels les jeunes ont été ou se sont soumis émergent comme indicateurs d'un mode de vie instable. Guy (nom fictif), 20 ans, présente à ce titre un cas-limite :

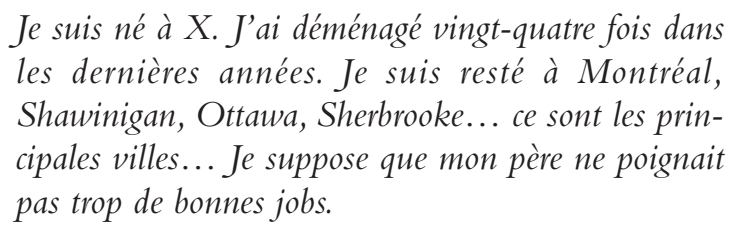

Ces déménagements constants limitent la capacité des jeunes de tisser des liens d'amitié durables à l'extérieur de leurs familles. Sans lieu ou espace d'ancrage et d'identification sociale définis, ils développent des rapports fragiles face à l'espace de vie physique et social qu'ils occupent.

\section{L'école}

La majorité des jeunes ont fréquenté uniquement des écoles françaises. Les niveaux scolaires varient parmi les jeunes 
Reflets

interviewés mais aucun n'a échappé au décrochage à un moment ou l'autre dans les deux dernières années. Ces constats se comparent aux recherches de Kurtz, Jarvis et Kurtz (1991) et Kufeldt et Burrows (1994). Les quatre jeunes de 20 et 21 ans n'ont pas terminé le secondaire; parmi ces quatre, deux sont inscrits à des cours par correspondance en $11^{\mathrm{e}}$ année et en CPO (cours préuniversitaire de l'Ontario). Par contre, les cinq jeunes de 16 à 18 ans accusent peu de retard dans leurs études, sauf pour le jeune homme de 17 ans qui n'a qu'une $8^{\text {e }}$ année. Une des deux femmes de 18 ans a entamé son programme de CPO tandis que l'autre a complété sa $10^{\mathrm{e}}$ année. Les deux femmes de 16 ans ont terminé la $9^{\mathrm{e}}$ et la $10^{\mathrm{e}}$ année. Ces femmes sont donc relativement plus avancées dans leurs études que les hommes. La plupart des jeunes adoptent des stratégies de scolarisation alternative (école alternative ou cours par correspondance) afin de poursuivre leurs études et semblent assez satisfaits de cette méthode d'apprentissage.

\section{Travail et revenu}

Tous les jeunes ont eu de l'expérience de travail surtout dans des emplois précaires, temporaires ou à temps partiel. Ces emplois se trouvent au bas de l'échelle salariale et dans le secteur des services (serveuse dans un fast food, vendeuse de vêtements, pelletage de neige...).

Le système d'aide sociale semble représenter un filet de sécurité important pour ces jeunes. Tous (sauf une répondante qui habite chez ses parents et occupe un emploi à temps partiel) sont prestataires d'aide sociale ou sont à faire la demande.

\section{Raisons expliquant l'itinérance}

Les facteurs explicatifs de l'itinérance se sont articulés autour de deux pôles: le départ du foyer parental et la descente vers la rue. Il est à remarquer que dans ces deux scénarios, la question du choix d'un mode de vie ne se pose pas pour les jeunes. Dans le premier scénario, celui du départ du foyer parental, il y a soit rejet par les parents, soit une fuite volontaire des jeunes qui ne voient pas leur sort s'améliorer dans leur milieu familial. Les parents étaient, 
"C'est souvent un ensemble de facteurs qui a occasionné le départ ou le rejet des jeunes...» selon les jeunes, soit abusifs ou très restrictifs. C'est souvent un ensemble de facteurs qui a occasionné le départ ou le rejet des jeunes: violence verbale et physique, manque d'espace à la maison, rejet d'un ou des parents à la suite d'une action non appréciée (confrontation au sujet de l'alcoolisme du père, non respect des règlements - sévères — des parents, conflits avec la loi) et la pauvreté matérielle du milieu familial.

La plupart des jeunes ont eu recours à d'autres stratégies avant de devenir itinérants: cohabiter avec des amis ou des connaissances, vivre dans une maison de chambre (tout en étant trop pauvres pour manger), tenter une réconciliation familiale (sans succès). Les facteurs qui ont mené les jeunes à l'itinérance sont complexes, agissent la plupart du temps de concert, reflètent des histoires de vie où les liens socio-familiaux avaient déjà été fragilisés et l'insertion à la filière scolarité-travail menacée. Les situations problèmes que rencontrent les jeunes interviewés dans la rue sont, en ce sens, en continuité avec leurs histoires de vie et leur situation d'itinérance.

\section{Résulats de la recherche: analyse interprétative}

Cette analyse se développe en liaison avec deux composantes: dans un premier temps, la question de la place occupée par l'identité franco-ontarienne dans les situations d'itinérance des jeunes interviewés sera abordée; dans un deuxième temps, les mécanismes de l'exclusion sociale des jeunes interviewés seront analysés afin de dégager leurs stratégies de survie et de sortie de l'itinérance.

\section{La place occupée parl'identitéfranco-ontarienne}

Les jeunes interviewés sont ambivalents et même incertains dans leur façon de décrire leur identité. 
Reflets

Moi, je suis Ontarien, mais je ne sais pas si je m'ap-

pellerais franco ou quoi.Je ne suis pas certain. (Richard)

Parce que je suis née au Québec et que j'ai vécu également dans les deux provinces, je me considère canadienne... je ne sais pas... je ne perds plus mon temps à m'identifier. (Simone)

Je sais que je suis anglophone et francophone mais tout le monde me dit que je parle aussi bien le français que l'anglais. Mais je me considère comme un anglophone avec une deuxième langue qui est le français. (Eric)

La question de leur identité fait plutôt place à celle de la langue d'usage. Pour les jeunes interviewés, la question de la langue française prend beaucoup d'importance étant donné l'utilité d'être bilingue à Ottawa-Carleton.

Quand tu es bilingue, ça t'aide à avoir une job parce que tous les gens qui parlent français en Ontario ont une job. (Geneviève)

Je ne veux pas perdre ma langue parce que je sais que c'est bon pour les affaires. (Denise)

Tu peux trouver plus d'emplois et parler à plus de gens parce que tu es bilingue. Tu te fais plus d'amis comme ça. (Eric)

Ainsi, l'identité franco-ontarienne des jeunes interviewés n'est pas très bien définie; en revanche lorsqu'ils parlent de la langue française, ils entretiennent un discours plus clair, quoique plutôt centré sur les avantages du bilinguisme. Ces avantages qu'ils perçoivent ne sont pas sans lien avec leur situation socio-économique d'exclusion; en effet, l'accent placé sur les avantages du bilinguisme plutôt que sur ceux de la capacité de parler le français dénote probablement une stratégie d'intégration au monde du travail, comme les citations précédentes semblent l'indiquer. À cet égard, 
"Leur situation

d'exclusion socioéconomique semble les pousser à adopter une identité bilingue plutôt que francoontarienne comme stratégie d'intégration afin d'avoir plus de chance d'obtenir un emploi.» les attitudes des jeunes interviewés different peu de celles des jeunes Franco-Wellandais qui habitent le sud de l'Ontario:

(ces jeunes sont) fier/ères d'être Canadiens/nes Français/es ou Franco-Ontariens/nes, mais (que) c'est surtout leur bilinguisme qu'ils ou elles revendiquent et valorisent. Ils et elles disent posséder un double héritage, anglophone et francophone, et que cela leur aidera à trouver un meilleur emploi. (Cardinal, Lapointe et Thériault, 1990:135)

Il semble donc que l'identité franco-ontarienne n'a pas beaucoup d'importance pour les jeunes sans abri qui ont été interviewés: elle n'apparaît clairement ni au niveau de leur choix identitaire ni en relation avec leurs stratégies d'intégration sociale. Ainsi, si l'école alternative en français se profile comme une stratégie d'intégration, elle ne semble pas être rattachée à une quelconque identité franco-ontarienne. Leur situation d'exclusion socio-économique semble les pousser à adopter une identité bilingue plutôt que franco-ontarienne comme stratégie d'intégration afin d'avoir plus de chance d'obtenir un emploi. De plus, les intervenants dans le milieu des organismes communautaires qui oeuvrent auprès des jeunes sans abri ne parlent habituellement pas le français; au moment de la recherche, les employés de l'abri d'urgence ne pouvaient même pas identifier les jeunes francophones qui bénéficiaient de leurs services.

\section{Les stratégies de survie et les stratégies de sortie}

Les stratégies de survie se divisent en deux catégories. Les stratégies personnelles des jeunes se centrent beaucoup sur des gens qui vont les aider et qu'ils peuvent aider en retour:

Ils (les gens dans la rue) t'aident parce qu'un jour toi tu pourras les aider quand ils n'auront rien. Si tu $n$ 'as pas de cigarette, il $y$ a toujours quelqu'un qui en a et qui va t'en donner... (Eric) 
Reflets

"Ce silence des jeunes sur les stratégies de sortie liées aux programmes et aux services plus formels vient souligner l'absence de politiques sociales visant l'intégration des jeunes sans abri.»
Ils nouent donc des relations avec d'autres qui sont aux prises avec des problèmes semblables. Ceci peut cependant entraîner un prolongement de la situation de l'itinérance et un endiguement dans l'exclusion; ça permet à tout le moins de résoudre des problèmes ou des conflits à court terme en établissant des liens valorisants.

Les stratégies de survie liées aux organismes et aux services s'appuient sur l'aide sociale, les services d'urgence et certaines haltesaccueil. Ce sont des relations utilitaires que les jeunes entretiennent avec ces institutions; celles-ci agissent comme filet de sécurité sociale sans offrir de pistes d'intégration ou même de possibilités de liens sociaux.

Les stratégies de sorties des jeunes visent leur intégration dans des relations socio-familiales et des filières d'intégration scolaritétravail. Ce sont soit leurs relations avec d'autres, soit des efforts individuels qu'ils ont déployés qui sont le plus fréquemment évoqués. Ce qui est le plus frappant au sujet des stratégies de sortie dans les discours des jeunes interviewés, c'est l'absence quasi totale de stratégies d'intégration qui s'appuient sur des ressources institutionnelles, l'accent étant plutôt mis sur des stratégies personnelles de sortie. Ce silence des jeunes sur les stratégies de sortie liées aux programmes et aux services plus formels vient souligner l'absence de politiques sociales visant l'intégration des jeunes sans abri. La plupart des interventions menées par le Département des services sociaux de la Municipalité régionale d'OttawaCarleton pour les jeunes sans abri durant la dernière décennie se résument à l'établissement de services d'urgence (abris, soupes populaires, etc.) et donc à la gestion de la crise personnelle. Les jeunes interviewés veulent terminer leurs études, éviter des relations violentes, trouver un emploi, cohabiter avec des amies et amis ou améliorer leurs relations avec ceux-ci ou avec leurs parents. Ainsi, un chevauchement s'opère entre les deux types de stratégies de sorties personnelles, en ce sens qu'elles agissent toutes les deux sur les piliers principaux du lien social, c'est-à-dire les liens socio-familiaux et l'intégration au monde du travail (filière scolarité-travail). Cependant, il ressort clairement que les programmes et les services destinés aux jeunes sans abri ne s'appuient pas sur des interventions facilitant l'intégration par les 
voies de la scolarité et de l'emploi. Deux conséquences importantes en découlent. Premièrement, même si certaines haltesaccueil constituent des espaces de création de liens sociaux pour les jeunes interviewés, ces programmes ne sont pas accompagnés de stratégies institutionnelles visant l'intégration à l'école ou au travail. Ainsi, les liens sociaux qui sont créés ont tendance à se former au sein du groupe des exclus, ce qui risque d'autant plus d'endiguer les jeunes dans l'exclusion. Deuxièmement, comme c'est le chacun pour soi qui prime au niveau de l'intégration aux filières de scolarité-emploi pour les jeunes interviewés, plusieurs d'entre eux ne réussissent pas leur intégration. En ce sens, plus ces filières leur échappent, plus leur période d'exclusion s'en trouvera prolongée.

\section{Implications pour l'intervention}

La plupart des interventions et des recherches pratiquées auprès des jeunes sans abri ne font que répondre à leur situation d'urgence ou cherchent à individualiser leurs problèmes. Elles considèrent ces gens-là comme des cas-problèmes. Elles perçoivent les jeunes commes des objets d'analyse et d'intervention dépourvus de discours et d'actions normalisants sur leur situation, et sur qui il s'avère nécessaire d'apposer les catégories de à risque ou en besoin. La recherche effectuée ici interpelle les intervenantes et intervenants, autant que les chercheures et chercheurs, à dépasser leur manière d'appréhender la réalité des jeunes sans abri.

\section{Reconceptualiser l'intervention auprès des jeunes sans abri}

Le modèle d'intervention de la Halte-accueil du Bureau des services à la jeunesse d'Ottawa-Carleton (BSJ) relève d'approches psycho-sociales et centrées sur la tâche. Ce modèle est axé sur l'expertise de l'intervention basée sur le renforcement du potentiel des jeunes pour produire des changements chez la clientèle et modifier le fonctionnement individuel. L'approche peut aussi 
"...car un jeune sans abri n'est pas qu'une personne à problèmes qui se retrouve sans toit, sans parents, sans amis, etc.»
"Il s'avère nécessaire de construire une approche d'intervention axée sur les liens sociaux et qui vise la réinsertion à la scolarité et à l'emploi.» être considérée comme centrée sur la tâche dans le sens où la visée de l'intervention assure la centralité de la clientèle et ses valeurs intrinsèques (Auclair et Lampron,1987:334-337).

Cependant, ces approches ne cherchent pas à intervenir sur les systèmes extérieurs à la réalité immédiate des jeunes ni à critiquer l'idéologie dominante. Elles ont comme effet d'isoler les jeunes, de les identifier comme les responsables de leur propre sort, plaçant ainsi le fardeau des sorties de crise sur leurs épaules, et contribuant à leur victimisation. Cette manière d'individualiser les problèmes des jeunes sans abri s'insère dans une vision de l'intervention qui se définit en fonction du cas par cas et qui se construit autour de relations d'aide qui misent sur la thérapie ou le counseling individuel. Même si les approches d'interventions du BSJ sont centrées sur la clientèle et cherchent donc à libérer chez les jeunes une capacité déjà existante d'auto-actualisation (Rowe,1986:416), celles-ci sont tellement axées sur l'individu qu'elles voilent et font même disparaitre le sens du monde au sein duquel s'insèrent les jeunes. S'établissent en ce sens des relations d'aide formelles qui misent sur l'expertise et les techniques d'intervention professionnelles. Conceptualiser ainsi l'intervention auprès des jeunes sans abri tend à nier la complexité de leurs problèmes car un jeune sans abri n'est pas qu'une personne à problèmes qui se retrouve sans toit, sans parents, sans amis, etc. Ils sont aussi des jeunes qui envisagent le monde à leur manière, qui tissent des liens sociaux, qui cherchent des moyens de «'en sortir», bref qui se débrouillent selon leur situation socio-économique et les facteurs d'exclusion sociale qui tendent à les isoler.

Les méthodes d'intervention auprès des jeunes doivent donc être abordées autrement. Cette tâche n'est pas simple car elle sous-entend un déplacement dans la manière d'approcher le problème social de l'itinérance des jeunes. Ce qui est proposé ici ne constitue ni plus ni moins qu'un bris de regard sur les jeunes sans abri: non plus objets d'intervention mais sujets d'action en lutte contre l'exclusion sociale qui pèse sur eux. Non plus des jeunes à problèmes mais bien des jeunes aux prises avec des problèmes d'intégration. Il s'avère nécessaire de construire une approche d'intervention axée sur les liens sociaux et qui vise la réinsertion 
à la scolarité et à l'emploi. Une telle approche requiert un dépassement des interventions parcellaires effectuées par les institutions d'aide et par catégories de problème et appelle la réduction de l'émiettement des responsabilités entre les dispositifs d'intervention sociale (Strohl et Xiberras,1992:192). Ainsi nous devrions nous attarder à des façons d'intervenir qui permettent aux jeunes de renouer les liens sociaux et reprendre contrôle de leurs vies.

\section{Modèled'intervention}

Un exemple de modèle d'intervention auprès de jeunes aux prises avec des problèmes d'intégration permet de mieux cerner les possibilités d'action sociale avec les jeunes sans abri. Des intervenantes américaines (Glover,1993; Pittman et Fleming, 1991) ont développé le Modèle de développement jeunesse (Youth Development Model) sur lequel peuvent se greffer des pratiques d'intervention qui tiennent compte à la fois du lien social et de l'intégration des jeunes à la filière scolarité-travail. Même si les auteurs indiquent que ce modèle s'applique surtout aux jeunes qui se retrouvent dans des situations de vulnérabilité sociale, il peut tout aussi facilement porter sur l'itinérance des jeunes. Les auteures et auteurs insistent sur le besoin de mobiliser un ensemble de programmes et d'activités qui puissent répondre à la complexité des intérêts et besoins des jeunes. L'application de ce modèle à l'échelon local, dans la région d'Ottawa-Carleton, a pris forme récemment dans un projet intitulé The Door/La porte qui est en voie d'élaboration. Ce projet est calqué sur des expériences d'intervention américaines qui ont cherché à structurer une gamme d'activités et de programmes tenant compte des champs de l'éducation, de la santé, des arts visuels et de la scène, des loisirs, de l'emploi, etc., autour du concept d'une halte-accueil (Barrette,1994).Ainsi, on tente de considérer la complexité de la vie des jeunes dans une perspective d'une approche holistique. L'application de ce modèle dans la réalité s'instaure donc en opposition aux approches d'intervention plus cliniques dont l'action thérapeutique se déploie sur un social à problème. Elle propose aussi une façon de surmonter (et non de remplacer, 
Reflets

car là n'est pas son objectif) les services d'urgence parcellaires et partiels qui ont été mis en oeuvre dans ces dernières années dans la région d'Ottawa-Carleton (soupes populaires, abris d'urgence, banques alimentaires, etc). La figure 1 illustre à quel point le Modèle de développement jeunesse de The Door/La porte cherche à offrir aux jeunes de nombreux points d'appui, tout en constituant une rupture avec le modèle d'intervention clinique de la Halte-accueil du BSJ.

Figure 1

Modèles d'intervention auprès des jeunes sans abri

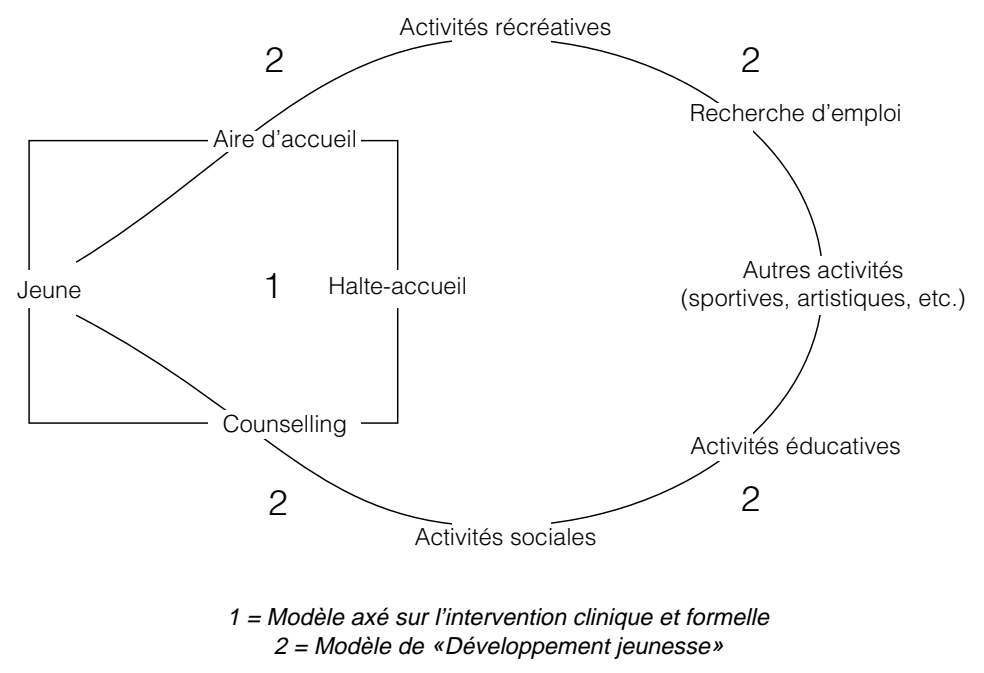

Dans cette illustration, de nombreux programmes et activités concernent directement le lien social à renouer et reflètent les stratégies de survie et de sortie des jeunes interviewés; on les retrouve au sein des activités sportives, sociales ou artistiques de la halte-accueil. De plus, des programmes pouvant mener à l'intégration à la filière scolarité-emploi se profilent à travers les activités éducatives et de préparation à l'emploi. L'ensemble des 
activités sert à situer les jeunes selon la complexité de leurs rapports à leur situation socio-économique et relationnelle; il se profile un mouvement qui fait réapparaitre les jeunes sans abri comme des sujets d'action, des citoyennes et citoyens qui s'inscrivent dans un projet démocratique d'inclusion, de reconnaissance et de participation.

\section{L'intervention auprès desjeunes francophones}

L'intervention doit aussi tenir compte de l'identité socio-culturelle des jeunes francophones. Comme mentionné précédemment, l'absence d'espaces sociaux francophones pour les jeunes sans abri contribue à l'émergence de stratégies d'intégration dont les points d'appuis (programmes, amies et amis, intervenantes et intervenants, institutions) sont situés dans un contexte anglophone, favorisant ainsi leur assimilation socio-culturelle. Face au contexte de la pauvreté et de l'exclusion des jeunes francophones sans

"...nous ne pouvons

pas apporter un

élément de solution

qui soit uniquement scolaire car il ne saura jamais être... suffisamment englobant pour tenir compte de la réalité complexe de l'itinérance..." abri, nous ne pouvons pas apporter un élément de solution qui soit uniquement scolaire car il ne saura jamais être (ni ne devrait-il l'être) suffisamment englobant pour tenir compte de la réalité complexe de l'itinérance, de la pauvreté, de l'exclusion sociale des jeunes. Ce constat interpelle les leaders, les chefs de file, les éminences grises de la communauté franco-ontarienne qui font de la lutte scolaire une véritable panacée. Le phénomène de la modernisation de la communauté franco-ontarienne ne doit plus continuer à exclure celles et ceux qui en sont les laissés pour compte (Welch,1994: 12). Cette pratique a l'effet pervers de décourager bon nombre de francophones à maintenir une identité franco-ontarienne.

Comme élément de solution pour les jeunes francophones sans abri, nous proposons une stratégie d'intervention double qui tienne compte des réalités, des forces et des enjeux pour les services sociaux et la communauté franco-ontarienne. Nous suggérons que des pressions soient exercées auprès de certains organismes communautaires, auprès du Département des services sociaux de la MROC, et auprès de diverses instances politiques. Cette stratégie viserait à établir des espaces sociaux francophones au sein de programmes et services habituellement considérés anglophones 
Reflets

"Nous proposons aussi que soient mis sur pied des programmes en français conçus et gérés par et pour des francophones...» ou bilingues. Nous proposons aussi que soient mis sur pied des programmes en français conçus et gérés par et pour des francophones à des niveaux stratégiques de l'intégration sociale des jeunes sans abri. Par exemple, des maisons de transition qui se positionnent entre les situations d'itinérance des jeunes et la vie indépendante en logement privé ou social constitueraient des points d'appui francophones importants pour les jeunes au niveau de leurs stratégies de survie; elles permettraient de recréer des situations de vie communes en français et donc de donner aux jeunes francophones des lieux pour nouer des liens sociaux, pour les soutenir et les encourager dans leurs stratégies d'intégration à la filière scolarité-travail.

\section{Conclusion}

Quelques rapports et études ont déjà abordé la question des services et des programmes en français dans le domaine des services sociaux et de la santé d'une façon directe (Association canadienne pour la santé mentale, 1988; Conseil de planification sociale, 1993; Gagnon,1994; Molgat, 1994 et 1994). Ces études démontrent le besoin de poursuivre le développement de programmes et services en français dans les champs des services sociaux et de la santé. La recherche dont il a été question dans cet article a tenté d'élaborer une manière d'aborder la question de l'itinérance et de l'identité franco-ontarienne des jeunes sans abri qui soit différente de celle qui pose ces mêmes jeunes comme une population à problèmes et les francophones comme des à côté de l'intervention. La méthode de recherche privilégiée proposait en ce sens de donner la parole aux jeunes, de faire émerger leurs perceptions et leurs actions, bref de les considérer comme des sujets de recherche qui se construisent des manières d'être et d'agir à partir de leur situation. L'exclusion sociale qui traverse les discours de tous les jeunes interviewés a servi de perspective d'analyse pour cette recherche. Ces jeunes sont aux prises avec une double exclusion: d'abord comme sans abri et ensuite comme francophones. L'analyse de leurs stratégies 
de survie et de sortie les a fait apparaître comme des acteurs sociaux qui se construisent, dans la mesure du possible, des manières de s'opposer à l'exclusion. Ils cherchent à se faire entendre afin de recevoir une aide qui ne les exclut pas davantage mais qui leur permet de négocier l'assistance et de la refuser ou de l'accepter selon les circonstances. La reconceptualisation de l'intervention auprès des jeunes sans abri passe par une façon plus holistique d'approcher le problème social de l'itinérance des jeunes. L'intervention devrait chercher à renouer les liens sociaux et favoriser la réinsertion des jeunes à la scolarité et à l'emploi afin de les appuyer dans leurs luttes contre l'exclusion. Dans le sens où elle servirait à situer les jeunes selon la complexité de leurs rapports à leur situation socio-économique et relationnelle, l'application du Modèle de développement jeunesse permettrait de faire ressortir la qualité de sujet d'action des jeunes et empêcherait la problématisation de leurs expériences.

L'intervention auprès des jeunes francophones sans abri devrait se situer à deux niveaux. D'abord elle devrait insister auprès des instances politiques et bureaucratiques pour que soient modifiés des programmes et services qui existent déjà afin que les réalités socio-économiques des francophones soient prises en considération. Ensuite, elle devrait se développer au sein même de la communauté et appeler les francophones à mettre sur pied des points d'appui autres que scolaires pour les jeunes sans abri. Ces programmes seraient conçus et gérés par et pour des francophones et donneraient aux jeunes d'autres raisons matérielles et affectives pour maintenir et développer une appartenance socioculturelle franco-ontarienne.

Quant aux recherches futures sur les jeunes sans abri, elles devraient tenir compte, dans leurs interprétations du problème de l'itinérance, de la richesse des discours des jeunes. Ces discours peuvent éclairer les logiques d'action des jeunes et la valeur sociale de ces dernières. Poser ainsi un regard analytique sur les jeunes sans abri en tant que sujets d'action dans le monde, c'est participer à un projet démocratique de rapprochement entre le problème de l'itinérance et le social de tous les jours. 
Reflets

\section{Bibliographie}

ARIÈS, Philippe (1973). L'enfant et la vie familiale sous l'Ancien Régime, Paris, Éditions du Seuil.

AUCLAIR, René et Christiane LAMPRON (1987). «Approche intégrée: une innovation dans la dispensation des services sociaux", Service social, vol. 36, no 2-3, pp.315-341.

ASSOCIATION CANADIENNE POUR LA SANTÉ MENTALE (1988). Quand j'suis malade j'suis pas bilingue: Rapport sur la planification des services de santé mentale en français pour les adultes de 16 à 65 ans, Ottawa.

BARETTE, Danielle (1994). The Door - La porte Update (memo presented to the Commissioner of the Social Services Department), Ottawa, Regional Municipality of Ottawa-Carleton.

BEAUD, Jean-Pierre (1992). «L'échantillonnage», dans Benoît Gauthier, dir. Recherche sociale: de la problématique à la collecte de données, Sainte-Foy, Presses de l'Université du Québec, pp.195-226.

BÉGIN, Patricia (1992) (1989). Les sans-abri au Canada, Ottawa, Bibliothèque du Parlement, Service de recherche, Division des affaires politiques et sociales.

BERNIER, Léon, Anne MORISSETTE et Gilles ROY (1992). "La fugue chez les adolescents: épisode d'un parcours biographique», Apprentissage et socialisation, vol. 15, no 1, pp.63-72.

BOURDIEU, Pierre (1980). Questions de sociologie, Paris, Éditions de Minuit.

BRENNAN,Tim, David HUIZINGA et Delbert ELLIOT (1978). The Social Psychology of Runaways, Toronto, Lexington Books.

CARDINAL, Linda, Jean LAPOINTE et J.Y. THÉRIAULT (1990). «La jeunesse ontaroise, une jeunesse moderne? Le cas des jeunes francowellandais/es», dans Dany Laveault, Jean-Marie Joly et Lionel Desjarlais, dir. Regards sur le jeune Franco-Ontarien: Actes du colloque, Ottawa: Centre franco-ontarien de ressources pédagogiques, pp.149-156.

CASTEL, Robert (1991). «De l'indigence à l'exclusion: la désaffiliation», dans Jacques Donzelot, dir. Face à l'exclusion: le modèle français, Paris, Éditions Esprit, pp.137-168.

COMITÉ CONSULTATIF DU MINIST RE SUR L'ANNÉE INTERNATIONALE DU LOGEMENT DES SANS-ABRI (1988). Plus qu'un simple toit: pour mettre fin au problème des sansabri en Ontario, Toronto, ministère du Logement de l'Ontario.

COMMUNITY TASK FORCE ON HOMELESS/STREETYOUTH (1992). Support Services to Homeless/Street Youth in Ottawa-Carleton: A Needs Assessment and Plan for Action, Ottawa.

CONSEIL DE PLANIFICATION SOCIALE D'OTTAWA-CARLETON (1989). Enquête sur les jeunes en matière d'hébergement à Ottawa-Carleton, Ottawa.

CONSEIL DE PLANIFICATION SOCIALE D'OTTAWA-CARLETON (1993). Étude sur les besoins en professionnel(le)s francophones dans les domaines de la santé et des services sociaux, Ottawa.

CONSEIL NATIONAL DU BIEN-ÊTRE SOCIAL (1993). Revenus de bien-être social — 1992, Ottawa, ministère des Approvisionnements et Services Canada.

CÔTÉ, Marguerite Michelle (1991). Les jeunes de la rue, Montréal, Éditions Liber.

DESLAURIERS, Jean-Pierre (1991). Recherche qualitative: guide pratique, Montréal, McGraw-Hill.

GAGNON, Brigitte (1993). Rapport d'étude: Accès des services en français à l'assistance financière dans la région d'Ottawa-Carleton (préparé dans le cadre d'un stage de recherche-intervention à l'Association canadienne pour la santé mentale), Ottawa.

GLOVER, Julie (1993). What Can be Done to Promote Comprehensive Youth Development Within Youth Programs? 
GUTTON, Jean-Pierre (1974). «Au seizième siècle: les grandes options», La société et les pauvres en Europe, Vendôme, Presses Universitaires de France, pp.93-121

HUBER, Jacqueline (1987). Sans toit ni droits: des jeunes en difficulté, Paris, Éditons S.O.S.

KUFELDT, Kathleen et Barbara A. BURROWS (1994). Issues Affecting Public Policies and Services for Homeless Youth, Calgary, University of Calgary; St-John's, Memorial University of Newfoundland.

KURTZ, David, Sara JARVIS et Gail KURTZ (1991). "Problems of Homeless Youths: Empirical Findings and Human Services Issues”, Social Work, vol. 36, no 4,pp. 309-314.

LALONDE, Lise (1993). «Repères pour des représentations contemporaines de l'itinérance et de l'intervention auprès des itinérants à Montréal», Intervention, vol. 94, mars, pp.52-67.

LAMONTAGNE, Yves et al. (1987). La jeunesse québécoise et le phénomène des sans-abri, Sillery, Presses de l'Université du Québec.

MAYER, Robert et Francine OUELLET (1991). Méthodologie de recherche pour les intervenants sociaux, Boucherville, Gaëtan Morin Éditeur.

MEYER, Philippe (1977). L'enfant et la raison d'État, Paris, Éditions du Seuil.

MOLGAT, Marc (1994). Des jeunes à l'écart du social? Histoires de jeunes francophones sans abri à OttawaCarleton, mémoire de maitrise déposé à l'École de service social de l'Université d'Ottawa, Ottawa.

MOLGAT, Marc (1994). Minoritaires et sans abri: des francophones en marge à Ottawa-Carleton Rapport sur les services en français dans les abris d'urgence à Ottawa-Carleton (préparé pour ActionLogement avec la collaboration de Nancy Gervais), septembre.

PITTMAN, Karen Johnson et Wanda FLEMING (1991). A New Vision: Promoting Youth Development, Washington, Academy for Educational Development.

ROBERTS, Albert (1981). Runaways and Non-Runaways in an American Suburb, New York, John Jay Press.

ROWE, William (1986). “Client-Centered Theory”, dans Francis Turner, dir. Social Work Treatment: Interlocking Theoritical Approaches, New York, Free Press, pp.407-431.

SIMARD, Pierre (1990). Le clochard de Montréal: une histoire à coucher dehors, Montréal, Éditions Saint-Martin.

STROHL, Hélène et Martine XIBERRAS (1992). Exclus et exclusions: connaître les populations, comprendre les processus - Rapport du groupe technique, présidé par Philippe Nasse, Paris, La Documentation Française.

URSEL, Jane (1992). Private Lives, Public Policy: 100 Years of State Intervention in the Family, Toronto, Women's Press.

WELCH, David (1994). «Les Franco-Ontariens: gagnants ou perdants?», Actes du Congrès de fondation du Regroupement des intervenantes et intervenants francophones en santé et en services sociaux de l'Ontario, Ottawa.

WELCH, David (1991-92). «Les luttes pour les écoles secondaires francoontariennes», Revue du Nouvel-Ontario, pp.13-14, pp.109-132.

WHITBECK, Les et Ronald SIMONS (1990). "The Victimization of Runaway and Homeless Adolescents", Youth and Society, vol. 22, no 1, pp.108125.

XIBERRAS, Martine (1993). Les théories de l'exclusion, Paris, Méridiens Klincksieck. 